VOL. 48 (1993) [303-311]

\title{
THE DUNFORD-PETTIS PROPERTY ON VECTOR-VALUED CONTINUOUS AND BOUNDED FUNCTIONS
}

\author{
Jose Aguayo and Jose Sanchez
}

\begin{abstract}
Let $X$ be a completely regular space, $E$ a Banach space, $C_{b}(X, E)$ the space of all continuous, bounded and $E$-valued functions defined on $X, M(X, \mathcal{L}(E, F))$ the space of all $\mathcal{L}(E, F)$-valued measures defined on the algebra generated by zero subsets of $X$. Weakly compact and $\beta_{0}$-continuous operators defined from $C_{b}(X, E)$ into a Banach space $F$ are represented by integrals with respect to $\mathcal{L}(E, F)$-valued measures. The strict Dunford-Pettis and the Dunford-Pettis properties are established on $\left(C_{b}(X, E), \beta_{2}\right)$, where $\beta_{2}$ denotes one of the strict topologies $\beta_{0}, \beta$ or $\beta_{1}$, when $E$ is a Schur space; the same properties are established on $\left(C_{b}(X, E), \beta_{0}\right)$, when $E$ is an $A M$-space or an $A L$-space.
\end{abstract}

\section{NOTATIONS AND DEFINITIONS}

Let $X$ be a completely regular space, $E$ a Banach space, and $C_{b}(X, E)$ the space of all continuous, bounded and $E$-valued functions defined on $X$ (if $E=\mathbb{R}$, then $\left.C_{b}(X, \mathbb{R})=C_{b}(X)\right)$. We use $B(X)(B a(X))$ to denote the smallest algebra ( $\sigma$-algebra) containing the zero-sets, the so-called Baire algebra (Baire $\sigma$-algebra), and $M(X)$ to denote the space of all zero-set regular measures defined on $B(X)$. We define $M\left(X, E^{\prime}\right)$ to be the space of all finitely additive vector measures $\mu: B(X) \rightarrow E^{\prime}$ such that

(1) for each $x \in E, \mu_{x}$, defined by $\mu_{x}(B)=\langle\mu(B), x\rangle$, is in $M(X)$

(2) $|\mu|(X)<\infty$, where $|\mu|(B)=\sup \left\{\left|\Sigma\left\langle\mu\left(B_{i}\right), s_{i}\right\rangle\right|:\left\{B_{i}\right\}\right.$ is a finite $B(X)$ partition of $B$ and $\left\{s_{\imath}\right\}$ is a finite collection from the unit ball of $E$ \}.

It is known that $C_{b}(X, E)^{\prime}=M\left(X, E^{\prime}\right)$.

For $B \in B(X), \mu \in M\left(X, E^{\prime}\right)$ and a $\mu$-measurable function $f$, we define $\int_{B} f d \mu=$ $\lim \Sigma \mu\left(B_{i}\right) f\left(x_{i}\right)$, where the limit is taken over the directed set of all finite $B(X)$ partitions of $B$ and $x_{i} \in B_{i}$.

Let $F$ be another Banach space. We define $M(X, \mathcal{L}(E, F))$ to be the space of all finitely additive vector measures $\mu: B a(X) \rightarrow \mathcal{L}(E, F)$ such that

(1) for each $x^{\prime} \in F^{\prime}, x^{\prime} \mu$, defined by $x^{\prime} \mu(B)=x^{\prime}(\mu(B))$, is in $M\left(X, E^{\prime}\right)$,

\section{Received 9 October 1992}

We are very grateful to Professor S.S. Khurana for several suggestions. This work was partially supported by FONDECYT, proyect 89-655 and Dirección de Investigación, Universidad de Concepción, proyect 91.12.21-1.

Copyright Clearance Centre, Inc. Serial-fee code: 0004-9729/93 \$A2.00+0.00. 
(2) $\|\mu\|(X)<\infty$, where for $B \in B(X),\|\mu\|(B)=\sup \left\{\left|x^{\prime} \mu\right|(B):\left\|x^{\prime}\right\| \leqslant\right.$ $1\}$.

Let $\mu \in M(X, \mathcal{L}(E, F))$ and let $f$ be a $\mu$-measurable function from $X$ into $E$. We say that $f$ is $\mu$-integrable in $B \in B(X)$ if

(i) for each $x^{\prime} \in F^{\prime}$, the integral $\int_{B} f d\left(x^{\prime} \mu\right)$ exists

(ii) there exists a vector in $F$, denoted by $\int_{B} f d \mu$, such that for all $x^{\prime} \in F^{\prime}$ we have $x^{\prime}\left(\int f d \mu\right)=\int f d\left(x^{\prime} \mu\right)$.

If $f$ is $\mu$-integrable over all $B$ in $B(X)$, we say that $f$ is $\mu$-integrable.

We shall pay careful attention [8] to three classes of Baire measures described as follows:

Let $\mu$ be a Baire measure. $\mu$ is called a $\sigma$-additive measure if $\mu\left(E_{n}\right) \rightarrow 0$ for every sequence $\left\{E_{n}\right\}$ in $B(X)$ such that $E_{n} \downarrow \emptyset . \mu$ is called $\tau$-additive if $\mu\left(E_{\alpha}\right) \rightarrow 0$ for every net $\left\{E_{\alpha}\right\}$ in $B(X)$ such that $E_{\alpha} \downarrow \emptyset . \mu$ is called tight if, given $\varepsilon>0$, there exists a compact subset $K$ of $X$ such that $|\mu|(X \backslash K)<\varepsilon$. It is known that each of these measures can be extended to $B a(X)[11]$.

We shall denote by $M_{\sigma}(X), M_{\tau}(X)$ and $M_{t}(X)$ the space of all $\sigma$-additive, $\tau$ additive and tight measures respectively. We shall understand for $M_{\sigma}\left(X, E^{\prime}\right)$ the space of all vector measures $\mu \in M\left(X, E^{\prime}\right)$ such that $\mu_{x} \in M_{\sigma}(X)$, for all $x \in E$. Similar meanings for $M_{\tau}\left(X, E^{\prime}\right)$ and $M_{t}\left(X, E^{\prime}\right)$. Finally, we shall understand by $M_{\sigma}(X, \mathcal{L}(E, F))$ the space of all vector measures $\mu \in M(X, \mathcal{L}(E, F))$ such that $y^{\prime} \mu: B(X) \rightarrow E^{\prime}$ belongs to $M_{\sigma}\left(X, E^{\prime}\right)$, for all $y^{\prime} \in F^{\prime}$. Similar meanings for $M_{\boldsymbol{r}}(X, \mathcal{L}(E, F))$ and $M_{t}(X, \mathcal{L}(E, F))$. Clearly, we have that $M_{t}(X, \mathcal{L}(E, F)) \subset$ $M_{r}(X, \mathcal{L}(E, F)) \subset M_{\sigma}(X, \mathcal{L}(E, F))$.

We shall define three locally convex topologies on $C_{b}(X, E)$, denoted by $\beta_{1}, \beta$ and $\beta_{0}$, as follows:

Let $\Omega$ and $\Omega_{1}$ be, respectively, the class of all compact and all zero sets in $\beta X \backslash X$, where $\beta X$ denotes the Stone-Cěch compactification of $X$. Let $Q \in \Omega\left(\Omega_{1}\right)$. We define $\beta_{Q}$ as the locally convex topology generated by the family of semi-norms $f \rightarrow\|f g\|$, where $g \in B_{Q}=\left\{h \in C_{b}(X): \widehat{h} \equiv 0\right.$ on $\left.Q\right\}$ ( $\widehat{h}$ denotes its extension to $\left.\beta X\right) . \beta\left(\beta_{1}\right)$ is the inductive limit of the topologies $\beta_{Q}$ as $Q$ ranges over $\Omega\left(\Omega_{1}\right) . \beta_{0}$ is defined as the finest locally convex topology which coincides on the norm bounded sets with the compact-open topology.

It is known that $\left(C_{b}(X, E), \beta_{i}\right)^{\prime}=M_{i}\left(X, E^{\prime}\right)$, where $\beta_{i}$ is any one of the above topologies.

The following characterisation of $\beta_{0}$-equicontinuous will be used [3].

LEMMA 1. A subset $H$ of $M_{t}\left(X, E^{\prime}\right)$ is $\beta_{0}$-equicontinuous if and only if

(a) $H$ is norm-bounded, and 
(b) for every $\varepsilon>0$ there exists a compact set $K \subset X$ such that $|\mu|(X \backslash K)<\varepsilon$ for all $\mu \in H$.

Recall that a linear operator $T$ from a topological vector space $A$ into another $B$ is weakly compact if it maps bounded subsets of $A$ into relatively weakly compact subsets of $B$.

Katsara and Liu [7] showed the following theorem:

THEOREM 2. Let $F$ be another Banach space. If $T$ is a continuous weakly compact operator from $C_{b}^{r c}(X, E)=\left\{f \in C_{b}(X, E): f(X)\right.$ is relatively compact in $E\}$ into $F$, then there exists a unique $m \in M(X, \mathcal{L}(E, F))$ such that

(1) every $f \in C_{b}^{r c}(X, E)$ is $m$-integrable and $T(f)=\int f d m$;

(2) $\|T\|=\|m\|(X)$;

(3) for every $x^{\prime} \in F$, we have $T^{\prime} x^{\prime}=x^{\prime} m$;

(4) for every bounded set $S$ in $E$, the set $V_{m S}=\left\{\sum m\left(G_{i}\right) s_{i}:\left\{G_{i}\right\}\right.$ is a finite $B$-partition of $\left.X, s_{i} \in S\right\}$ is relatively weakly compact.

Conversely, if $m \in M(X, \mathcal{L}(E, F))$ is such that (4) holds, then every $f$ in $C_{b}^{r c}(X, E)$ is $m$-integrable and the operator $T(f)=\int f d m$ is norm-continuous and weakly compact.

The following theorem is a characterisation of a $\beta_{0}$-continuous and weakly compact operator $T$ defined from $C_{b}^{r c}(X, E)$ into $F$. It is known that $C_{b}^{r c}(X, E)$ is $\beta_{0}$-dense in $\left(C_{b}(X, E), \beta_{0}\right)$.

THEOREM 3. Let $T$ be a weakly compact operator defined from $C_{b}^{r c}(X, E)$ into $F$. The following statements are equivalent:

(1) $T$ is $\beta_{0}$-continuous.

(2) $T_{\mid B}$ is compact-open-continuous, where $B$ is the unit ball of $C_{b}^{r c}(X, E)$.

(3) $(\forall \varepsilon>0)(\exists K \subset X, K$ compact $) \quad\left(\left|x^{\prime} m\right|(X \backslash K)<\varepsilon\right.$ uniformly for $\left\|x^{\prime}\right\|$ $\leqslant 1)$, where $m$ is the associated vector measure given in the above theorem.

Proof: Since $\beta_{0}$ is the finest locally convex topology agreeing with the compactopen topology on bounded subsets of $C_{b}(X, E)$, we have (1) $\Leftrightarrow(2)$.

$(3) \Rightarrow(1)$. Let $\left\{f_{\alpha}\right\}$ be a compact-open null convergent net. Hence, for a given $\varepsilon>0$, there exists a compact subset $K$ of $X$ such that $\|m\|(X \backslash K)=$ $\sup \left\{\left|x^{\prime} m\right|(X \backslash K):\left\|x^{\prime}\right\| \leqslant 1, x^{\prime} \in F^{\prime}\right\}<\varepsilon$, which implies that $\left|x^{\prime} m\right|(X \backslash K)<\varepsilon$ uniformly for $x^{\prime} \in F^{\prime},\left\|x^{\prime}\right\| \leqslant 1$. Therefore, $\left\{x^{\prime} m: x^{\prime} \in F^{\prime},\left\|x^{\prime}\right\| \leqslant 1\right\}$ is a $\beta_{0}$ equicontinuous subset of $M_{t}\left(X, E^{\prime}\right)$. Since $x^{\prime} m=T^{\prime} x^{\prime}$ and $\left(C_{b}^{r c}(X, E), \beta_{0}\right)^{\prime} \cong$ $M_{t}\left(X, E^{\prime}\right)$, we have $\left\{T^{\prime} x^{\prime}: x^{\prime} \in F^{\prime},\left\|x^{\prime}\right\| \leqslant 1\right\}$ is also $\beta_{0}$-equicontinuous.

Finally, since $\|T f\|=\sup \left\{\left|T^{\prime} x^{\prime}(f)\right|: x^{\prime} \in F^{\prime},\left\|x^{\prime}\right\| \leqslant 1\right\}$, we conclude that $T f_{\alpha} \rightarrow 0$ in norm. 
(1) $\Rightarrow$ (3). Since $\left\{T^{\prime} x^{\prime}: x^{\prime} \in F^{\prime},\left\|x^{\prime}\right\| \leqslant 1\right\}$ is $\beta_{0}$-equicontinuous is equivalent to $\left\{x^{\prime} m: x^{\prime} \in F^{\prime},\left\|x^{\prime}\right\| \leqslant 1\right\}$ is $\beta_{0}$-equicontinuous, the arguments are similar to these given above.

THEOREM 4. Let $T$ be a $\beta_{0}$-continuous and weakly compact linear operator defined on $C_{b}(X, E)$. Then there exists a unique $m \in M(X, \mathcal{L}(E, F))$ such that

(1) every $f \in C_{b}(X, E)$ is $m$-integrable and $T(f)=\int f d m$;

(2) $\|T\|=\|m\|(X)$;

(3) for every $x^{\prime} \in F$, we have $T^{\prime} x=x^{\prime} m$;

(4) for every bounded set $S$ in $E$, the set $V_{m S}=\left\{\sum m\left(G_{i}\right) s_{i}:\left\{G_{i}\right\}\right.$ is a finite $B$-partition of $\left.X, s_{i} \in S\right\}$ is relatively weakly compact.

Proof: Since $C_{b}^{r c}(X, E)$ is $\beta_{0}$-dense in $\left(C_{b}(X, E), \beta_{0}\right), T$ is the unique $\beta_{0}$ continuous extension of $T_{\left.\right|_{C_{b}^{r c}(X, E)}}$ to $C_{b}(X, E)$. By Theorem $3, T_{\left.\right|_{C_{b}^{r c}(X, E)}}$ has a unique vector measure $m$ associated to it which satisfies (1), (2), (3) and (4).

By the fact that if norm $T$ and its restriction are the same and their respective transposes coincide, we only have to prove that each $f \in C_{b}(X, E)$ is $m$-integrable and $T(f)=\int f d m$.

Let $f \in C_{b}(X, E)$; hence, by the definition of the topology $\beta_{0}$ and the density of $C_{b}^{r c}(X, E)$, there exists a net $\left\{f_{\alpha}\right\} \in C_{b}^{r c}(X, E)$ inside the ball of radius $\|f\|$ such that $f_{\alpha} \rightarrow f$ uniformly on compact subsets of $X$. Take any $G \in B a(X)$; since the dual of $\left(C_{b}(X, E), \beta_{0}\right)$ is $M_{t}\left(X, E^{\prime}\right)$, we have that, for any $x^{\prime} \in F^{\prime}, \int_{G} f d x^{\prime} m$ is defined. At the same time, it is not difficult to see that $\left\{\int_{G} f d m\right\}$ is a norm-Cauchy net in $F$ and then it is convergent to some $v_{G} \in F$.

We claim that $\int_{G} f_{\alpha} d x^{\prime} m \rightarrow \int_{G} f d x^{\prime} m$. In fact, given $\varepsilon>0$, there exists a compact subset $K$ of $X$, such that $\left|x^{\prime} m\right|(G \backslash K) \leqslant\left|x^{\prime} m\right|(X \backslash K)<\varepsilon$ uniformly for $\left\|x^{\prime}\right\| \leqslant 1$. Also, there exists $\alpha_{0}$ such that, for $\alpha \geqslant \alpha_{0}$,

$$
\sup \left\{\left\|f_{\alpha}(x)-f(x)\right\|: x \in K\right\}<\varepsilon .
$$

The claim follows from

$$
\begin{aligned}
\left|\int_{G}\left(f_{\alpha}-f\right) d x^{\prime} m\right| & \leqslant \int_{G}\left\|f_{\alpha}-f\right\| d\left|x^{\prime} m\right| \\
& \leqslant \int_{G \cap K}\left\|f_{\alpha}-f\right\| d\left|x^{\prime} m\right|+\int_{G \backslash K}\left\|f_{\alpha}-f\right\| d\left|x^{\prime} m\right| .
\end{aligned}
$$

Since $x^{\prime}\left(\int_{G} f_{\alpha} d m\right)=\int_{G} f_{\alpha} d x^{\prime} m \rightarrow x^{\prime}\left(v_{G}\right)$ and $\int_{G} f_{\alpha} d x^{\prime} m \rightarrow \int_{G} f d x^{\prime} m$, we conclude that $\int_{G} f d x^{\prime} m=x^{\prime}\left(v_{G}\right)$. Defining $v_{G}=\int_{G} f d m$, we have, for each $G \in B a(X)$, $\int_{G} f_{\alpha} d m \rightarrow \int_{G} f d m$; in particular, $T\left(f_{\alpha}\right)=\int_{X} f_{\alpha} d m \rightarrow \int_{X} f d m$. Therefore, by the continuity of $T$, we have $\int_{X} f d m=T(f)$. 
We shall adopt the following definitions:

A locally convex space $E$ is said to have the Dunford-Pettis (respectively strict Dunford-Pettis) property if for any Banach space $F$, any weakly compact and continuous operator $T$ from $E$ into $F$ transforms absolutely convex weakly compact subsets (respectively weakly Cauchy sequences) of $E$ into relatively compact subsets (respectively convergent ones) of $F[6]$. It is known that both definitions are equivalent in Banach spaces.

The next theorem was proved in [9] and it establishes some relation between the Dunford-Pettis (D-P) and the strict Dunford- Pettis (strict D-P) properties.

THEOREM 5. If $E^{\prime}$, the topological dual of $E$, has a $\sigma$-compact dense subset in the $\sigma\left(E^{\prime}, E\right)$-topology and $E$ has the strict $D$-P property then $E$ has the $D$-P property.

\section{The strict Dunford-Pettis and the Dunford-Pettis properties}

In this section, we shall discuss, first, the strict D-P and the D-P properties on $\left(C_{b}(X, E), \beta_{i}\right)$ with $E$ a Schur space. After that, we shall discuss the same properties on $\left(C_{b}(X, E), \beta_{0}\right)$ with some special space $E$.

It is known that in $C_{b}(X, E), \beta_{i}$-bounded subsets coincide with normed-bounded subsets.

Let $T$ be an $F$-valued, weakly compact and $\beta_{i}$-continuous operator defined on $C_{b}(X, E)$. Since the strict topologies are coarser than the norm topology and have the same bounded subsets, we have that $T$ is also $\|$. $\|$-continuous and weakly compact. Therefore by Theorem $2, T_{\left.\right|_{C_{b}^{r c}(X, E)}}$ has an associated measure $m$ of $M(X, \mathcal{L}(E, F))$.

LEMma 6 . Let $T$ be an operator as above. Then there exists a nonnegative finite real-valued, $\sigma$-additive measure $\mu$ such that, for any $\varepsilon>0$, there exists $\delta>0$, so that for each $A \in B a(X)$ with $\mu(A)<\delta$, we have $\left|x^{\prime} m\right|(A)<\varepsilon$, uniformly for $\left\|x^{\prime}\right\| \leqslant 1$.

Proof: It is easy to see that each $x^{\prime} m$ in $M_{i}\left(X, E^{\prime}\right)$ can be extended to $B a(X)$ and that $M_{i}\left(X, E^{\prime}\right)$ is $\|$.$\| -closed in M\left(X, E^{\prime}\right)$.

Since $T$ is weakly compact, we have that $T^{\prime}: F^{\prime} \rightarrow C_{b}(X, E)^{\prime}=M\left(X, E^{\prime}\right)$ is weakly compact. Also, by the $\beta_{i}$-continuity of $T$, we have that $T^{\prime} x^{\prime} \in M_{i}\left(X, E^{\prime}\right)$. Therefore, if $B_{F^{\prime}}$ denotes the unit ball of $F^{\prime}$, then $\overline{T^{\prime}\left(B_{F^{\prime}}\right)} \|^{\|\cdot\|}$ is convex and weakly closed contained in $M_{i}\left(X, E^{\prime}\right)$. Now, since $\sigma\left(M_{i}\left(X, E^{\prime}\right), M_{i}\left(X, E^{\prime}\right)^{\prime}\right)$ coincides with the induced $\sigma\left(M\left(X, E^{\prime}\right), M\left(X, E^{\prime}\right)^{\prime}\right)$ on $M_{i}\left(X, E^{\prime}\right)$, we have that $T^{\prime}\left(B_{F^{\prime}}\right)$ is relatively weakly compact in $M_{i}\left(X, E^{\prime}\right)$.

Thus, $\left\{\left|x^{\prime} m\right|:\left\|x^{\prime}\right\| \leqslant 1\right\}$ is uniformly $\sigma$-additive and then there exists a nonnegative finite real-valued $\sigma$-additive measure $\mu$ such that for any $\varepsilon>0$, there exists $\delta>0$, so that for each $A \in B a(X)$ with $\mu(A)<\delta$, we have $\left|x^{\prime} m\right|(A)<\varepsilon$, uniformly for $\left\|x^{\prime}\right\| \leqslant 1[4]$. 
The measure $\mu$, given in the above theorem, is called a control measure of $\left\{\left|x^{\prime} m\right|:\left\|x^{\prime}\right\| \leqslant 1\right\}$.

A Banach space $E$ is a Schur space if weakly convergent sequences of $E$ are norm convergent on it.

THEOREM 7. If $E$ is a Schur space, then $\left(C_{b}(X, E), \beta_{i}\right)$ has the strict D-P property.

ProOF: Let $T$ be a weakly compact and $\beta_{i}$-continuous operator defined from $C_{b}(X, E)$ into a Banach space $F$. We shall denote by $m$ its associated vector measure.

Since $T$ is weakly compact and, in particular, $\beta_{1}$-continuous, we have that $\left\{\left|x^{\prime} m\right|:\left\|x^{\prime}\right\| \leqslant 1\right\}$ has a control measure $\mu$ ([Lemma 6]). Let $\left\{f_{n}\right\}$ be a weakly Cauchy sequence in $\left(C_{b}(X, E), \beta_{i}\right)$; hence $\left\{f_{n}\right\}$ is uniformly bounded and, for each $x \in X,\left\{f_{n}(x)\right\}$ is a weakly Cauchy sequence in $E$ (this follows from the fact that $\delta_{x} \otimes e^{\prime} \in M_{i}\left(X, E^{\prime}\right)$, where $\delta_{x} \otimes e^{\prime}(f)=e^{\prime}(f(x))$ and $\left.e^{\prime} \in E\right)$. Using the fact that $E$ is a Schur space, for each $x \in X,\left\{f_{n}(x)\right\}$ is norm-Cauchy. By Egoroff's Theorem, given $\varepsilon>0$, there exists a compact subset $K$ of $X$, such that $\mu(X \backslash K)<\varepsilon$ and $\left\{\left\|f_{n}\right\|\right\}$ converges uniformly on $K$. Now, since

$$
\left\|T\left(f_{n}-f_{m}\right)\right\|=\sup \left\{\left|x^{\prime} T\left(f_{n}-f_{m}\right)\right|:\left\|x^{\prime}\right\| \leqslant 1\right\}=\sup \left\{\left|\int f d x^{\prime} m\right|:\left\|x^{\prime}\right\| \leqslant 1\right\}
$$

and

$$
\left|\int f d x^{\prime} m\right| \leqslant \int_{K}\|f\| d\left|x^{\prime} m\right|+\int_{X \backslash K}\|f\| d\left|x^{\prime} m\right|<\varepsilon\left|x^{\prime} m\right|(K)+2 M \varepsilon
$$

uniformly for $\left\|x^{\prime}\right\| \leqslant 1$, where $M$ is the uniform bound of $\left\{f_{n}\right\}$, we have that $\left\{T\left(f_{n}\right)\right\}$ is convergent in $F$. This proves the theorem.

TheOREM 8. If $E$ is a Schur space and $X$ is $\sigma$-compact, then $\left(C_{b}(X, E), \beta_{i}\right)$ has the Dunford-Pettis property.

Proof: The proof is exactly the same as in Theorem $3.2[1]$.

Lemma 9. If $Y$ is a compact space, then $\left(C_{b}(X, C(Y)), \beta_{0}\right)$ is $\beta_{0}$-homeomorphic to $\left(C_{b}(X \times Y), \beta_{0}\right)$. Consequently, $\left(C_{b}(X, C(Y)), \beta_{0}\right)$ has both properties, the strict and the Dunford-Pettis properties [9].

Proof: The function $\Psi: C_{b}(X, C(Y)) \rightarrow C_{b}(X \times Y)$, define by $\Psi(f)(x, y)=$ $f(x)(y)$, is one-one and onto with inverse function $\Psi: C_{b}(X \times Y) \rightarrow C_{b}(X, C(Y))$ defined by $\Psi(g): X \rightarrow C(Y)$ where $\Psi(g)(x)(y)=g(x, y)$.

Note that $|\Psi(f)(x, y)|=|f(x)(y)| \leqslant\|f(x)\|$ which implies $\Psi$ is continuous in the $\beta_{0}$-topology.

On the other hand, if $\left\{f_{\alpha}\right\}$ is a net in $C_{b}(X \times Y)$, converging to 0 in the $\beta_{0}$ topology, and $K$ is a compact subset of $X$, then, by the compactness of $Y, f_{\alpha} \rightarrow 0$ uniformly on $K \times Y$; therefore $\Phi\left(f_{\alpha}\right) \rightarrow 0$ uniformly on $K$. 
An $A M$-space is a Banach lattice which satisfies the axiom $\|\sup \{x, y\}\|=$ $\sup \{\|x\|,\|y\|\}$. It is known that every $A M$-space $E$ with unit is isomorphic to $C(K)$, where $K$ is some compact space [10].

THEOREM 10. If $E$ is a $A M$-space, then $\left(C_{b}(X, E), \beta_{0}\right)$ has both properties, the strict and the Dunford-Pettis properties.

Proof: It follows from Lemma 9 and the fact that every $A M$-space is isomorphic to a $C(Y)$, for some compact space $Y$.

An $A L$-space is a Banach lattice which satisfies $\|x+y\|=\|x\|+\|y\|$ for $x \geqslant 0$, $y \geqslant 0$. It is known that every $A L$-space is isomorphic to $L^{1}(\mu)$, where $\mu$ is a Radon measure defined on a locally compact space.

Theorem 11. If $E$ is an $A L$-space, then $\left(C_{b}(X, E), \beta_{0}\right)$ has the strict DunfordPettis Property.

Proof: By the above remark, it is enough to prove that $C_{b}\left(X, L^{1}\right)$ has the strict Dunford-Pettis Property. Let $T$ be a weakly compact and $\beta_{0}$-continuous linear operator from $C_{b}\left(X, L^{1}\right)$ into a Banach space $F$ and let $m$ be its associated vector measure. Take an arbitrary weakly Cauchy sequence $\left\{f_{n}\right\}$ in $C_{b}\left(X, L^{1}\right)$ and denote by $M$ its uniform bound.

By Theorem 3, given $\varepsilon>0$, there exists a compact subset $K$ of $X$ such that $\left|x^{\prime} m\right|(X \backslash K)<\varepsilon / 4 M$, uniformly for $x^{\prime} \in F^{\prime},\left\|x^{\prime}\right\| \leqslant 1$.

We claim that $\left\{f_{\left.n\right|_{K}}\right\}$ is weakly Cauchy in $\left(C\left(K, L^{1}\right),\|\|.\right)$. In fact, let $\lambda$ be a continuous linear functional on $C\left(K, L^{1}\right)$ and let us define $\lambda_{1}: C_{b}\left(X, L^{1}\right) \rightarrow \mathbb{R}$ by $\lambda_{1}(f)=\lambda\left(f_{\left.\right|_{K}}\right)$. Clearly, $\lambda_{1}$ is $\|$.$\| and \beta_{0}$-continuous and then, $\lambda\left(f_{\left.n\right|_{K}}-f_{\left.m\right|_{K}}\right)=$ $\lambda_{1}\left(f_{n}-f_{m}\right) \rightarrow 0$ as $n, m \rightarrow \infty$.

Now, we define $L: C\left(K, L^{1}\right) \rightarrow F$ by $L(f)=\int_{K} f d m . L$ is weakly compact and $\|\cdot\|$-continuous. Therefore, since $C\left(K, L^{1}\right)$ has the strict Dunford-Pettis or simply the Dunford-Pettis property [2], we have that, for the $\varepsilon>0$ given above, there exists $N \in \mathbb{N}$ such that for $n, m \geqslant N$,

$$
\left\|L\left(f_{n}-f_{m}\right)\right\|=\left\|\int_{K}\left(f_{n}-f_{m}\right) d m\right\|<\varepsilon / 2
$$

and then

$$
\left|\int_{K}\left(f_{n}-f_{m}\right) d x^{\prime} m\right|<\varepsilon / 2 \text { uniformly for }\left\|x^{\prime}\right\| \leqslant 1
$$

Now, let $n, m \geqslant N$. Since

$$
\begin{aligned}
\left\|T\left(f_{n}-f_{m}\right)\right\| & =\sup \left\{\left|x^{\prime}\left(T\left(f_{n}-f_{m}\right)\right)\right|: x^{\prime} \in F,\left\|x^{\prime}\right\| \leqslant 1\right\} \\
& =\sup \left\{\left|\int\left(f_{n}-f_{m}\right) d x^{\prime} m\right|: x^{\prime} \in F,\left\|x^{\prime}\right\| \leqslant 1\right\},
\end{aligned}
$$


we have

$$
\begin{aligned}
\left|\int\left(f_{n}-f_{m}\right) d x^{\prime} m\right| \leqslant & \left|\int_{K}\left(f_{n}-f_{m}\right) d x^{\prime} m\right|+\int_{X \backslash K}\left\|f_{n}-f_{m}\right\| d\left|x^{\prime} m\right| \\
& <\varepsilon / 2+2 M(\varepsilon / 4 M)=\varepsilon
\end{aligned}
$$

uniformly for $\left\|x^{\prime}\right\| \leqslant 1$. Therefore,

$$
\left\|T\left(f_{n}-f_{m}\right)\right\|=\sup \left\{\left|\int\left(f_{n}-f_{m}\right) d x^{\prime} m\right|: x^{\prime} \in F,\left\|x^{\prime}\right\| \leqslant 1\right\}<\varepsilon
$$

for $n, m \geqslant N$.

This proves the theorem.

THEOREM 12. If $E$ is an $A L$-space, then $\left(C_{b}(X, E), \beta_{0}\right)$ has the Dunford-Pettis property.

Proof: By the isomorphism between $E$ and $L^{1}$, it is enough to assume that $E=L^{1}$.

We shall first suppose that $X$ has a $\sigma$-compact dense subset $X$. Then, developing the same argument given in [9, Theorem 3, p.363], we prove that $\left(M_{t}\left(X, L^{1}\right), w^{*}\right)$ contains a $\sigma$-compact dense. Therefore, by Theorem 5 and Theorem 11 we conclude that $\left(C_{b}\left(X, L^{1}\right), \beta_{0}\right)$ has the Dunford-Pettis property.

Suppose now that $X$ is not necessarily as above and let $T$ be a weakly compact, $\beta_{0}$-continuous operator defined from $C_{b}\left(X, L^{1}\right)$ into $F$ with associated vector measure $m$.

By Theorem 3, given $\varepsilon=1 / n$, there exists a compact subset $K_{n}$ of $X$ such that $\left|x^{\prime} m\right|\left(X \backslash K_{n}\right)<1 / n$, uniformly for $x^{\prime} \in F^{\prime},\left\|x^{\prime}\right\| \leqslant 1$. Defining $X_{0}$ as the closure of $\bigcup K_{n}$, we have that $X_{0}$ is a $\sigma$-compact subset of $X$ and $\left|x^{\prime} m\right|\left(X \backslash X_{0}\right)=0$, uniformly for $x^{\prime} \in F^{\prime},\left\|x^{\prime}\right\| \leqslant 1$.

We define $L: C_{b}\left(X_{0}, L^{1}\right) \rightarrow F$ by $L(f)=\int_{X_{0}} f d m$. We claim that $L$ is weakly compact and $\|\cdot\|$-continuous. By Katsara [7], it is enough to prove that, for all bounded $S$ in $E$, the sets $V_{S}$ and $V_{0_{S}}=\left\{\Sigma m\left(G_{i}\right) s_{i}:\left\{G_{i}\right\}\right.$ is a finite $B$-partition of $\left.X, s_{i} \in S\right\}$ are equal. In fact, we note first that, for any $s \in E,\|s\| \leqslant 1,\left|x^{\prime} m\left(X \backslash X_{0}\right) s\right| \leqslant$ $\left|x^{\prime} m\right|\left(X \backslash X_{0}\right)=0$ and then $\left\|m\left(X \backslash X_{0}\right) s\right\|=0$, which implies that $m\left(X \backslash X_{0}\right) \equiv 0$. By the same argument, we show that, for any $G \in B(X), m\left(G \cap X \backslash X_{0}\right) \equiv 0$. Thus,

$m(G) s=m\left(\left(G \cap X \backslash X_{0}\right) \cup\left(G \cap X_{0}\right)\right) s=m\left(G \cap X \backslash X_{0}\right) s+m\left(G \cap X_{0}\right) s=m\left(G \cap X_{0}\right) s$

and then $V_{S} \subset V_{0_{S}}$. On the other hand, let $\Sigma m\left(G_{i}\right) s_{i} \in V_{0_{S}}$, where $X_{0}=\bigcup G_{i}$ and $s_{i} \in S$. Hence, taking the $B(X)$-partition $G_{1}, \ldots, G_{n}, X \backslash X_{0}$ of $X$ and any $s \in S$, we have that $\Sigma m\left(G_{i}\right) s_{i}=\Sigma m\left(G_{i}\right) s_{i}+m\left(X \backslash X_{0}\right) s \in V_{S}$. 
Now, since $V_{S}$ is relatively weakly compact in $F$, so is $V_{0_{S}}$. Then $L$ is weakly compact and \|.\|-continuous [Theorem 4].

The operator $\phi: C_{b}\left(X, L^{1}\right) \rightarrow C_{b}\left(X_{0}, L^{1}\right)$ defined by $\phi(f)=f_{\mid X_{0}}$ is clearly $\beta_{0^{-}}$ continuous, since $X_{0}$ is closed. Therefore, $\phi$ is weak-weak continuous. Also, $T=L_{0} \phi$, since $m\left(X \backslash X_{0}\right) \equiv 0$. Therefore, if $B$ is an absolutely convex, weakly compact subset of $C_{b}\left(X, L^{1}\right)$, then $\phi(B)$ is an absolutely convex and relatively weakly compact subset of $C_{b}\left(X_{0}, L^{1}\right)$. Since $\left(C_{b}\left(X, L^{1}\right), \beta_{0}\right)$ has the D-P property, $L(\phi(B))$ is relatively compact in $F$, and since $T(B)=L(\phi(B))$, we have that $T$ transforms absolutely convex and weakly compact subsets of $C_{b}\left(X, L^{1}\right)$ into compact subsets of $F$. This proves the theorem.

\section{REFERENCES}

[1] J. Aguayo and J. Sanchez, 'Separable measures and The Dunford-Pettis property', Bull. Austral. Math. Soc. 43 (1991), 423-428.

[2] J. Bourgain, 'On the Dunford-Pettis property', Proc. Amer. Math. Soc. 81 (1981), 256-272.

[3] S. Choo, 'Strict topologies on space of continuous vector-valued functions', Canad. J. Math. 31 (1979), 890-898.

[4] J. Diestel and J. Uhl, Vector measures, Survey Number 15 (American Mathematical Society, Providence, 1977).

[5] R.A. Fontenot, 'Strict topologies for vector-valued functions', Canad. J. Math. 26 (1974), 831-853.

[6] A. Grothendieck, 'Sur les applications linéaires faiblement compact d'espaces du type $C(K)^{\prime}$, Canad. J. Math. 5 (1953), 129-173.

[7] A. Katsaras and B. Liu, 'Integral representations of weakly compact operator', Pacific J. Math. 56 (1975), 547-556.

[8] S.S. Khurana, 'Topologies on spaces of vector-valued continuous functions', Trans. Amer. Math. Soc. 241 (1978), 195-211.

[9] S.S. Khurana, 'Dunford-Pettis property', J. Math. Anal. Appl. 65 (1978), 361-364.

[10] H. Schaefer, Banach lattices and positive operators (Springer-Verlag, Berlin, Heidelberg, New York, 1974).

[11] R. Wheeler, 'A survey of Baire measures and strict topologies', Exposition Math. 29 (1983), 97-190.

Departamento de Matemática

Facultad de Ciencias Físicas y Matemáticas Universidad de Concepción

Casilla 3-C

Concepción

Chile 